\title{
The near wall behavior of an impinging jet
}

\author{
Danielle R.S. Guerra ${ }^{\mathrm{a}}$, Jian $\mathrm{Su}^{\mathrm{b}}$, Atila P. Silva Freire ${ }^{\mathrm{a}, *}$ \\ ${ }^{a}$ Mechanical Engineering Program (PEM/COPPE/UFRJ), C.P. 68503, 21945-970-Rio de Janeiro, Brazil \\ ${ }^{\mathrm{b}}$ Nuclear Engineering Program (PEN/COPPE/UFRJ), C.P. 68509, 21945-970-Rio de Janeiro, Brazil
}

Received 2 January 2005; received in revised form 31 January 2005

\begin{abstract}
The present work investigates the applicability of scaling log-laws to the turbulent impinging jet. Both, the velocity and the temperature fields are studied under this assumption. To validate the proposed expressions, a detailed experimental program was carried out based on thermal anemometry. The experiments were conducted for one nozzleto-plate spacing $(H / D=2.0)$ and Reynolds number of 35,000. A constant wall heat flux condition was achieved by conducting electricity through thin resistors that were placed beneath an aluminum disk. Measurements of local velocity and of temperature distributions are presented as well as longitudinal turbulence profiles. The mean temperature profiles were measured through thermocouples.
\end{abstract}

(c) 2005 Published by Elsevier Ltd.

Keywords: Impinging jet; Law of the wall; Hot-wire anemometry

\section{Introduction}

A turbulent jet impinging onto a surface is a very effective means to promote high rates of heat exchange. As an obvious implication, this geometrical arrangement has been extensively used in industrial processes that aim to achieve intense heating, cooling or drying rates. Typical applications are the tempering and shaping of glass, the annealing of plastic and metal sheets, the drying of textile and paper products, the deicing of aircraft systems and the cooling of heated components in gas turbine engines and electronic instruments.

\footnotetext{
* Corresponding author. Tel./fax: +55 2125627748.

E-mail addresses: daguerra@terra.com.br (D.R.S. Guerra), sujian@1mn.con.ufrj.br (J. Su), atila@mecanica.coppe.ufrj.br, atilafreire@gmail.com (A.P. Silva Freire).
}

Although the number of works devoted to this subject over the past thirty years has been consistently high, the recent times have witnessed a considerable increase in the number of published articles. Because of the high complexity of the flow structure resulting from an impinging jet, many new works have been published to study specific aspects of the problem. The generation of vortices at the entrance nozzle, the entrainment of fluid from the surrounding medium, the effects of the confining walls, the separation of flow, together with many other complicating factors make the delivery of a single and complete theory difficult to make. Thus, because of the several important aspects associated with the problem, studies have concentrated on characterizing different features of the phenomenon. This will be reviewed in the next section. However, we can anticipate here that most of the works that deal with theoretical analyses resort to asymptotic techniques and similarity concepts. 


$\begin{array}{ll}\text { Nomenclature } \\ A, B \quad \text { parameters in law of the wall } \\ D & \text { diameter of jet nozzle } \\ h & \text { heat transfer coefficient } \\ H & \text { distance between nozzle and impinging wall } \\ I & \text { electric current across resistances } \\ k & \text { Von Karman constant }(=0.41) \\ N_{u} & \text { Nusselt number } \\ q & \text { heat flux } \\ r & \text { radial distance from jet centerline } \\ T, t_{\tau} & \text { temperature, friction temperature } \\ T u[\%] & \left.\text { turbulent intensity (= } \sqrt{\overline{u^{\prime 2}}} / u\right) \\ U & \text { jet bulk velocity } \\ u, u^{\prime} & \text { local longitudinal mean velocity, local longi- } \\ & \text { tudinal velocity fluctuation }\end{array}$

\begin{tabular}{|c|c|}
\hline$u_{\tau}$ & friction velocity \\
\hline$V$ & voltage across resistances \\
\hline$x, y$ & Cartesian coordinates \\
\hline \multicolumn{2}{|c|}{ Greek symbols } \\
\hline$v$ & kinematic viscosity \\
\hline$\rho$ & density \\
\hline$\kappa$ & thermal diffusivity \\
\hline
\end{tabular}
Subscripts
$M, m$ maximum, minimum
$w, a w \quad$ wall condition, adiabatic wall condition
$v \quad$ total

For wall jets, we cannot positively say that the loglaw is a well established concept. In fact, several authors [1-4] have reported a large range of values for the loglaw constants. This certainly raises some important questions as to the validity on the use of the log-law for the estimation of surface friction and of the heat transfer coefficient.

Some researchers [5], however, have shown that for an oblique impinging jet the law of the wall can be observed for both the velocity and the temperature fields. More than that, these authors propose a functional behavior for the log-law parameters that resorts to a scaling procedure based on the stream-wise evolution of the flow characterized by its maximum velocity. In fact, Narasimha et al. [6] were one of the first to acknowledge that the traditional use of the nozzle diameter as the reference scaling for wall jet flows was not appropriate. They proposed a scaling length that took into consideration the flow evolution.

The purpose of the present work is to carry out further investigations on the scaling laws governing the motion of an orthogonal jet impinging onto a surface. For that matter, the law of the wall, for both the velocity and the temperature fields, will be investigated in detail. In fact, this is a necessary first step for the future investigation of some governing parameters on the skin-friction and on the heat transfer characteristics of a circular impinging jet on a heated flat plate. Two new expressions will be advanced for the law of the wall for the velocity and temperature fields that will resort to parametric arguments in the line of the works of Narasimha et al. [6] and of Özdemir and Whitelaw [5]. Measurements of local pressure, velocity and temperature distributions will be presented. We will also present data for the longitudinal turbulent intensities. The experiments were conducted for one nozzle-to-plate spacing and Reynolds number of 35,000 . A constant wall heat flux condition was achieved by conducting electricity through thin resistors that were placed beneath an aluminum disk. Temperature was measured using thermocouples.

At this point, it is important to make it clear to the reader that other authors have specifically studied the role of the scaling laws in wall jet flows. That is the case of the work of Wygnanski et al. [7] where the relevance of the wall to the evolution of the large coherent structures in the flow is studied. Here, in the impinging jet, the problem is further complicated by a deflection of the streamlines and by the presence of a stagnation point.

The recognition that for the wall jet the velocity profile does not exhibit the conventional law of the wall behavior is also verified in an article by Hammond [8]. Aiming at developing an analytic expression for the complete velocity field in the fully developed flow regime of a plane, turbulent wall, Hammond proposed to couple Spalding's single formula [9] for the inner layer with a sine function for the "wake component".

For turbulent flows, the mathematical description of the flow field is greatly complicated by the necessary specification of turbulence models that can capture all relevant characteristics of the problem. Frequently, turbulence models of the eddy viscosity type are used together with some heat transfer analogy consideration for the description of the temperature field (see e.g., [10-12]. This leads, for example, to serious difficulties at the stagnation point where the Reynolds analogy between eddy-diffusivity and eddy-viscosity breaks down. Indeed, when the equations of motion are integrated to the wall and the hypothesis of a constant turbulent Prandtl number is used, the calculated heat transfer rates at the stagnation point are observed to exceed by much their actual values. 
Despite the critics of many researchers, the use of wall functions to by-pass the difficulties involved with the modeling of low Reynolds number turbulence is still an attractive means to solve problems in a simple way. For instance, Cruz and Silva Freire $[13,14]$ have proposed an alternative approach where new wall functions are used to describe the velocity and temperature fields in the wall logarithmic region of a separating flow. As the stagnation point is approached, these functions reduce to power-law solutions recovering Stratford's solution. The paper of Cruz and Silva Freire resorted to Kaplun limits for an asymptotic representation of the velocity and temperature fields. Results were presented for the asymptotic structure of the flow and for the skin-friction coefficient and Stanton number at the wall.

\section{Short literature review}

For the wall jet, the first studies were severally limited by the lack of any sophisticated instrumentation. As a result, the first experiments were limited to measurements of mean velocities in the vicinity of the nozzle (see e.g., [15]). Sigalla [16] was the first to try to evaluate the skin-friction and also the first to measure the mean velocity at large distances from the nozzle. The development of the hot-wire anemometer in the 60's made it possible the development of much more detailed investigations.

Tailland and Mathieu [2] noticed that the rate of spread of a wall jet and the decay of its maximum velocity were dependent on the Reynolds number, a feature that is not observed in a free jet. That raised questions on the reason for such difference.

Scaling laws for wall jets were particularly studied by Patel [1], Ozarapoglu [3] and Irwin [4].

In relation to the impinging jet, the following are some of the notable references.

In the early 90's, Özdemir and Whitelaw [5] studied the problem emphasizing the large-scale transport of temperature by spatially coherent structures. These authors showed that an oblique impingement introduced vertical velocities that rendered the boundary layer equation inapplicable and resulted in a flow structure with strong azimuthally dependence. The large structures improved the transport of heat but led to an inactive zone near the vortex center.

Fox et al. [17] also studied the influence of vortical structures on the temperature field of jets. Depending on the distance of the jet nozzle to an adiabatic wall, secondary vortical structures were observed that could result in a region of lower wall temperature. Thus, it is the competition that is established between the vortex rings formed at the jet periphery and the secondary vor- tices resulting from the impingement that determines the wall temperature.

Cooper et al. [18] and Craft et al. [19] in two companion papers studied turbulent jets impinging orthogonally onto a plane surface. Cooper et al. reported an extensive set of measurements on the flow field; data for the mean velocity profile in the vicinity of the surface and also for the three Reynolds stress components lying in the $x-r$ plane were presented. These data were used by Craft et al. to examine the performance of four different turbulence models: the $\kappa-\varepsilon$ model and three-second order moment closures. The predictions obtained through the $\kappa-\varepsilon$ model and one second order moment closure resulted in far too high turbulence levels near the stagnation point. As such, they also resulted in too high heat transfer coefficients. Adaptations on the two other models led to much better predictions. None of the models, however, could successfully predict the Reynolds number effects on the flow. The authors concluded that this ought to be due to the two-equation eddy viscosity model that was adopted for all cases to span to the near wall sublayer.

The numerical simulation of impinging jets using a $\kappa-\varepsilon$ model was also performed by Knowles [20]. The author concluded that the Rodi and Malin corrections could not predict wall jet growth (see [20] for details).

Colucci and Viskanta [21] studied experimentally the effects of nozzle geometry on the local heat transfer coefficients of confined impinging jets. Low nozzle-to-plate gaps were considered in the Reynolds number range of 10,000-50,000. The results were compared with similar experiments for unconfined jets. An important conclusion was that the local heat transfer coefficients for confined jets are more sensitive to Reynolds number and nozzle-to-plate gaps than those for unconfined jets.

Dianat et al. [22] used a $\kappa-\varepsilon$ model and a modified second-moment closure to make velocity field predictions in the stagnation as well as in the jet region. The second-moment closure was modified to account for the influence of the wall in distorting the fluctuating pressure field away from it. With this modification, the damping of normal velocity fluctuations was well predicted.

The heat transfer in the flow of a cold, two-dimensional, vertical liquid jet impinging against a hot, horizontal, surface was given an approximate solution for the velocity and temperature fields by Shu and Wilkins [23]. The solution is valid for laminar flows and resorts to the hydrodynamic similarity solution of Watson (see the authors for details). The results were compared with a numerical realization of the flow.

Meola et al. [24] investigated the influence of shear layer dynamics on impingement heat transfer. Again, coherent structures and/or recirculation currents were observed to alter the distribution of heat transfer coefficients. Temperatures were measured with an infrared scanning radiometer whereas heat transfer coefficients 
were evaluated by the heated thin foil technique. The distribution of Nusselt number was discussed and a new explanation given for the local peak in the local Nusselt number.

Liu and Sullivan [25] investigated the heat transfer and flow structures in an excited circular impinging jet with a small nozzle-to-wall gap. Enhancement or reduction of the local heat transfer coefficient in the wall-jet region was shown to be achieved by exciting the impinging jet near to its natural frequency or sub-harmonics, respectively.

Nishino et al. [26] report the turbulence statistics in the stagnation region of an axisymmetric jet impinging vertically on a wall. They used particle-tracking velocimetry do measure the flow near the stagnation point and found that the turbulent normal stress of the axial component gave a substantial contribution to the increase in the static pressure near the wall. Turbulence was studied through an invariant map of the turbulent stress anisotropy. In the stagnation region, turbulence was close to an axisymmetric state.

The standard $\kappa-\varepsilon$ model together with the logarithmic law of the wall was applied by Ashforth-Frost and Jambunathan [27] to a semi-confined impinging jet; the nozzle-to-wall distance was two nozzle diameters and the Reynolds number 20,000. Laser-Doppler anemometry and liquid crystal thermography were used to determine velocity, turbulence and heat transfer data. In the developing wall jet, authors showed numerical heat transfer results to compare to within $20 \%$ of experimental data. However, at the stagnation point, heat transfer is over predicted by about $300 \%$. The authors attributed this discrepancy to failure of the wall function to conform to the physics of the flow.

San et al. [28] reported local measurements of Nusselt number for a confined impinging jet. The recirculation and the mixing effect on the heat transfer were investigated by varying the jet diameter, the surface heat flux, the Reynolds number and the surface heating width.

Knowles and Myszko [29] carried out turbulence measurements in a jet impinging onto a flat wall. Different nozzle-to-wall gaps were investigated. Measurements were conducted using hot-wire anemometry. Nozzle height was found to have a large effect on turbulence peak level for distances up to $r / d=4.5$; lower nozzleto-wall ratios caused an increase in peak level measured in all turbulent stresses in the stagnation region.

Kendoush in a previous work had derived analytical solutions for the convective heat and mass transfer predictions in a laminar jet impinging on a plane wall. However, his solution was shown to have a strong singularity at the stagnation point. The subsequent paper of 1998 [30] had, therefore, the objective of removing such singularity and find workable solutions for the problem. The results were compared with available experimental and numerical data.
Lee and Lee [31] experimentally studied the heat transfer behavior of a turbulent jet impinging on a wall with special attention to the stagnation region. For nozzle-to-wall ratios of $H / d=2.0$ the local Nusselt number variation with $r / d$ had two peaks and varied according to $R e^{0.5}$. For $H / d>6.0$, Nusselt number decreased monotonically with $r / d$. Reynolds number dependence was observed to increase as $H / d$ increases.

Confined impinging jets at low Reynolds numbers were experimentally studied by Baydar [32] for a single and a double jet. The author concludes that a sub-atmospheric region occurs on the impingement wall at nozzleto-wall gaps up to two and that there is a linkage between the sub-atmospheric region and the peak in the heat transfer coefficients.

In their next paper, Lee and Lee [33] studied experimentally the local heat transfer characteristics of an elliptic jet impinging on a heated flat plate for various nozzle aspect ratios. The temperature distributions on the heated plate were measured using a thermochromic liquid crystal thermometry with a digital image processing system. A smoke-wire technique was used to visualize the flow. For small nozzle-to-wall gap, as the aspect ratio of the elliptic jet increases, the heat transfer rate increased more than that for the circular jet in the stagnation region. This was attributed to the large entrainment and large scale mixing of the elliptic jet.

The control on the properties of an impinging jet by vortex pairing was investigated by Hwang et al. [34]. Two types of vortex control were performed: secondary shear flow and acoustic excitation. Some flow visualization, velocity and turbulence measurements were performed to understand the flow structure. Enhancement or reduction in heat transfer was observed by controlling the vortex pairing.

Guo and Wood [35] performed measurements in the vicinity of the stagnation point for an impinging jet with a very low free-stream turbulence level $(0.35 \%)$. The wall shear stress was measured with Preston tubes and Stanton probes. Due to the very small thickness of the boundary layer, $0.42 \mathrm{~mm}$, the sizes of the probes had to be kept to a minimum. In fact, the authors found that decreasing probe size the shear stress increased. In the end, the values found for the $0.11 \mathrm{~mm}$ Stanton probe were reported.

A flow direct numerical simulation was performed by Chung et al. [36] to investigate the velocity and the heat transfer characteristics of an unsteady impinging jet. These authors found that the unsteadiness in wall heat transfer is caused by the vortices emanating from the nozzle, and that these vortices significantly affect the stagnation heat transfer.

A finite element code that resorts to the streamline upwind method of Petrov-Galerkin and uses the $\kappa-\omega$ turbulence model was developed by Park et al. [37] to predict the flow and the heat transfer characteristics of 
two-dimensional confined impinging jets. Validation of the code was made against other codes and some measured mean velocity profiles.

The flow field and the local heat transfer for transitional impinging jets were characterized by Angioletti et al. [38] thorough particle image velocimetry and the naphthalene film technique. Local non-uniformities in heat transfer were explained through the destructive effects that large coherent structures created at the nozzle have upon impingement on the boundary layer.

Narayanan et al. [39] studied experimentally the flow field, surface pressure and the heat transfer rates for a submerged, turbulent, impinging jet. Two nozzle-to-surface spacing ratios were analyzed. Mean and turbulent flow properties were determined thorough a 1-D LDA system. Mean and RMS surface pressures were found with a piezoresistive transducer, surface temperatures were found using IR thermography. The results indicate that past impingement, and, irrespective of the nozzle spacing, locations of high streamwise fluctuating velocity variance occur in the wall jet. In particular, for the shorter of the two nozzle-to-surface spacing ratios, the authors found a good correlation between the location of a secondary peak in the heat transfer and the near wall streamwise fluctuating velocity variance.

The use of mesh screens to enhance the transfer of heat in impinging jets was investigated by Zhou and Lee [40]. Generally, it was observed that the screens modified the flow field leading to an increase in the local heat transfer coefficients.

\section{Experimental methods}

A drawing of the experimental apparatus is shown in Fig. 1. Air at $18.5^{\circ} \mathrm{C}$ is pumped through a centrifugal blower connected to a $1350 \mathrm{~mm}$ long pipe with $43.5 \mathrm{~mm}$ internal diameter. Inside the pipe, a honeycomb is fitted, constructed from drinking straws glued together; screens are also set in place. The jet is set to emerge from the circular nozzle with a bulk velocity of $12 \mathrm{~m} / \mathrm{s}$.

The impingement flat surface is made of a $3.7 \mathrm{~mm}$ thick aluminum circular sheet. This sheet has $840 \mathrm{~mm}$ in diameter and is laid over a plenum chamber as shown in Fig. 1. The plenum chamber is $20 \mathrm{~mm}$ height and

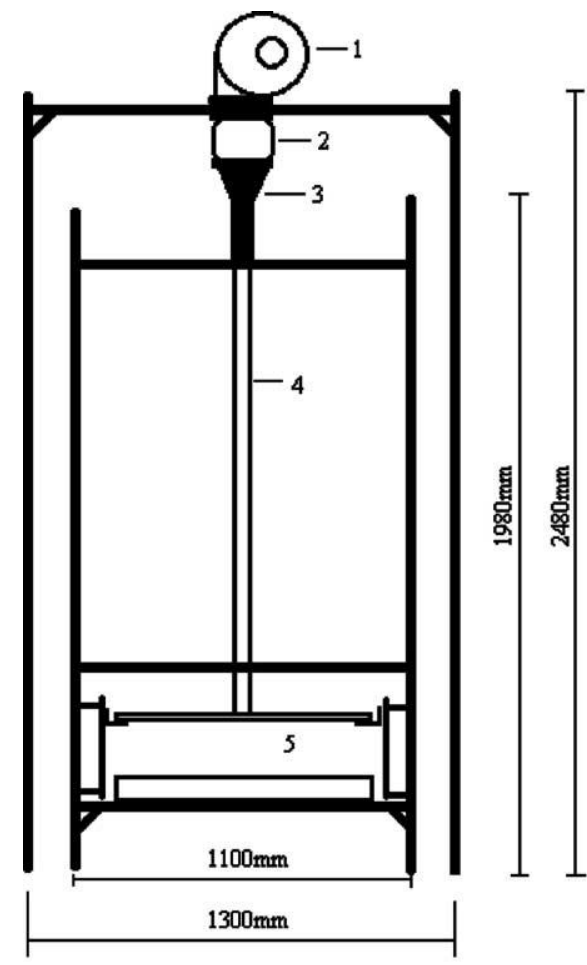

(a)

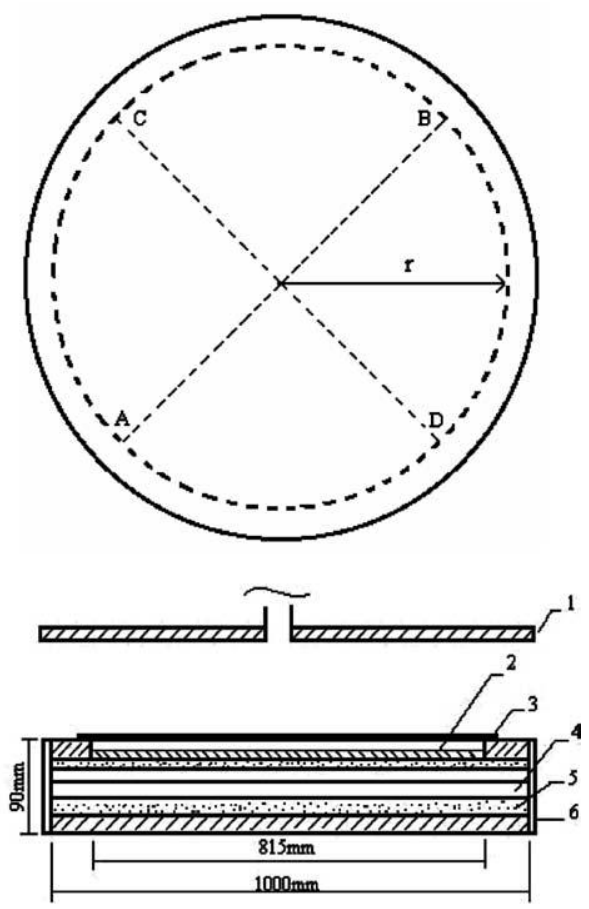

(b)

Fig. 1. Experimental apparatus. (a) Overall view: (1) centrifugal blower, (2) flexible section, (3) contraction, (4) pipe, and (5) test section. (b) Detail of test section and heating system: (1) confinement plate, (2) electrical resistance, (3) impingement plate, (4), (5) and (6) thermal isolation. 
$815 \mathrm{~mm}$ in diameter. At the bottom of the chamber a series of electrical resistances are placed to furnish a maximum of $4000 \mathrm{~W}$. The walls of the plenum were completely insulated from the ambient as shown in Fig. 1.

The controlled parameters in the experiments are the nozzle-to-plate spacing, the resistance heat flux and the stagnation pressure. At each test, the centerline of the jet is lined up with the center of the impingement surface

The temperature of the aluminum sheet was monitored through thermocouples. The readings of the thermocouples were routed to an AMD Athlon $+2000 \mathrm{MHz}$ personal computer via a Picolog acquisition system model TC-08.

Mean velocity profiles and turbulence intensity levels were obtained using a DANTEC hot-wire system series $56 \mathrm{~N}$. The boundary layer probe was of the type 55P15. A Pitot tube, an inclined manometer, and a computer controlled traverse gear system were also used. In getting the data, 10,000 samples were considered. The profiles were constructed from about 100 points. The mean temperature profiles were obtained through a chromelconstantan micro-thermocouple mounted on the same traverse gear system used for the hot-wire probe. The traverse gear system has $0.02 \mathrm{~mm}$ sensitivity.

An uncertainty analysis of the data was performed according to the procedure described in Kline [41]. The uncertainty associated with the velocity and temperature measurements were: $U=0.064 \mathrm{~m} / \mathrm{s}$ precision, 0 bias $(P=0.95) ; T=0.214{ }^{\circ} \mathrm{C}$ precision, 0 bias $(P=0.99)$.

The local convective heat transfer coefficient was calculated from

$h=\frac{q_{\mathrm{v}}}{T_{\mathrm{w}}-T_{\mathrm{aw}}}$,

where $T_{\mathrm{w}}$ and $T_{\mathrm{aw}}$ are wall temperature and adiabatic wall temperature of the stream. $q_{\mathrm{v}}$ is the total heat flux imposed through the electrical resistance and is given by $q_{\mathrm{v}}=\frac{4 I V}{\pi D^{2}}$.

The conduction heat flux was considered negligible and the radiation heat flux was calculated and was $2 \%$ of the total imposed heat flux. The local surface temperature was converted to the local Nusselt number defined as

$N_{u}=\frac{h D}{\kappa}$,

where $D$ is the jet exit diameter and $\kappa$ is the thermal conductivity of air.

To perform the experiments, the following procedure was applied. First, the flat plate was fitted with 27 pressure taps arranged in a cross formation. The readings of the pressure at these points were subsequently used to find the geometrical center of the jet; only when the pressure distribution was found to be completely symmetric the jet centerline was considered determined.

To find the adiabatic temperature, the electric current was turned off and the temperature recorded. Next, the resistors were turned on and the behavior of the wall temperature recorded. Normally, 6 control points were used at this stage. Only when the plate was observed to reach a steady state the blower was turned on. In the steady state condition, the wall temperature variation was within $1{ }^{\circ} \mathrm{C}$. Approximately $2.5 \mathrm{~h}$ are required to reach steady state conditions for each test run.

\section{Results}

The work will present results for the geometry defined by the aspect ratio $H / D=2.0$.

The radial pressure distribution on the impingement surface and the Nusselt number distribution are shown in Fig. 2. Pressure was made dimensionless with the dynamic pressure, $\rho U^{2} / 2$, where $\rho$ is the density of air and $U$ is the jet bulk velocity.

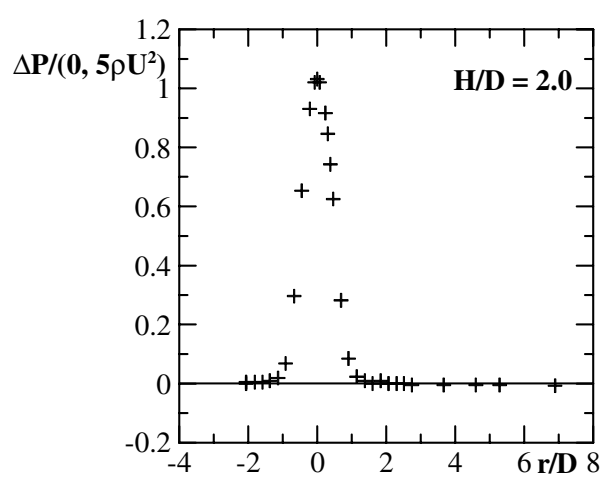

(a)

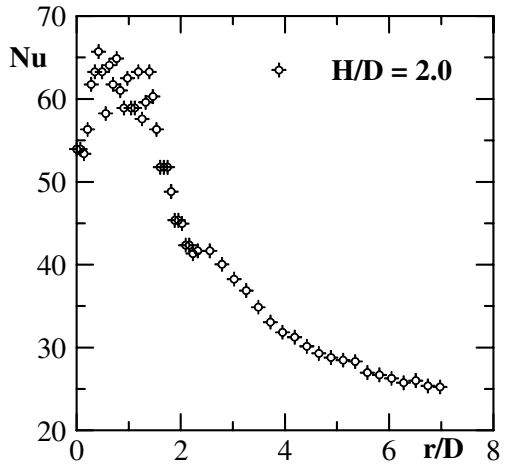

(b)

Fig. 2. (a) Radial pressure distributions of the jet and (b) Nusselt number distribution. 
For large $H / D$, nozzle-to-plate spacing rates, the Nusselt number is normally observed in literature to present a maximum at the stagnation point decreasing with increasing $r / D$. Here, this trend has been confirmed. The local heat transfer begins to increase from the stagnation point towards a first local peak position near $r / D \cong 0.5$. Then, Nusselt number decreases reaching a weak local maximum at about $r / D \cong 2.0$. After this point a second weak peak appears and then decreases.

To characterize the wall spreading of the jet, the radial components of the mean velocity, of the longitudinal turbulence intensity and of the mean temperature were examined at various radial positions. The velocity, longitudinal turbulent intensity and temperature profiles are shown in Figs. 3-5 in physical co-ordinates.
A study by Özdemir and Whitelaw [5] has shown that a Weibull distribution represents well some of the global features of the profile, such as the position of the maximum and of the outer inflection points, but is not an adequate approximation for the near wall region. For this region, they showed that semi-logarithmic relation can be used to model the inner equilibrium layer, so that one can write

$\frac{u}{u_{\tau}}=\frac{1}{\kappa} \ln \left(\frac{y u_{\tau}}{v}\right)+A$

where $u_{\tau}$ is the friction velocity and $\kappa$ is the von Karman constant.

The main contribution of Özdemir and Whitelaw [5] was to show that, for the impinging jet, the inner layer

(a)
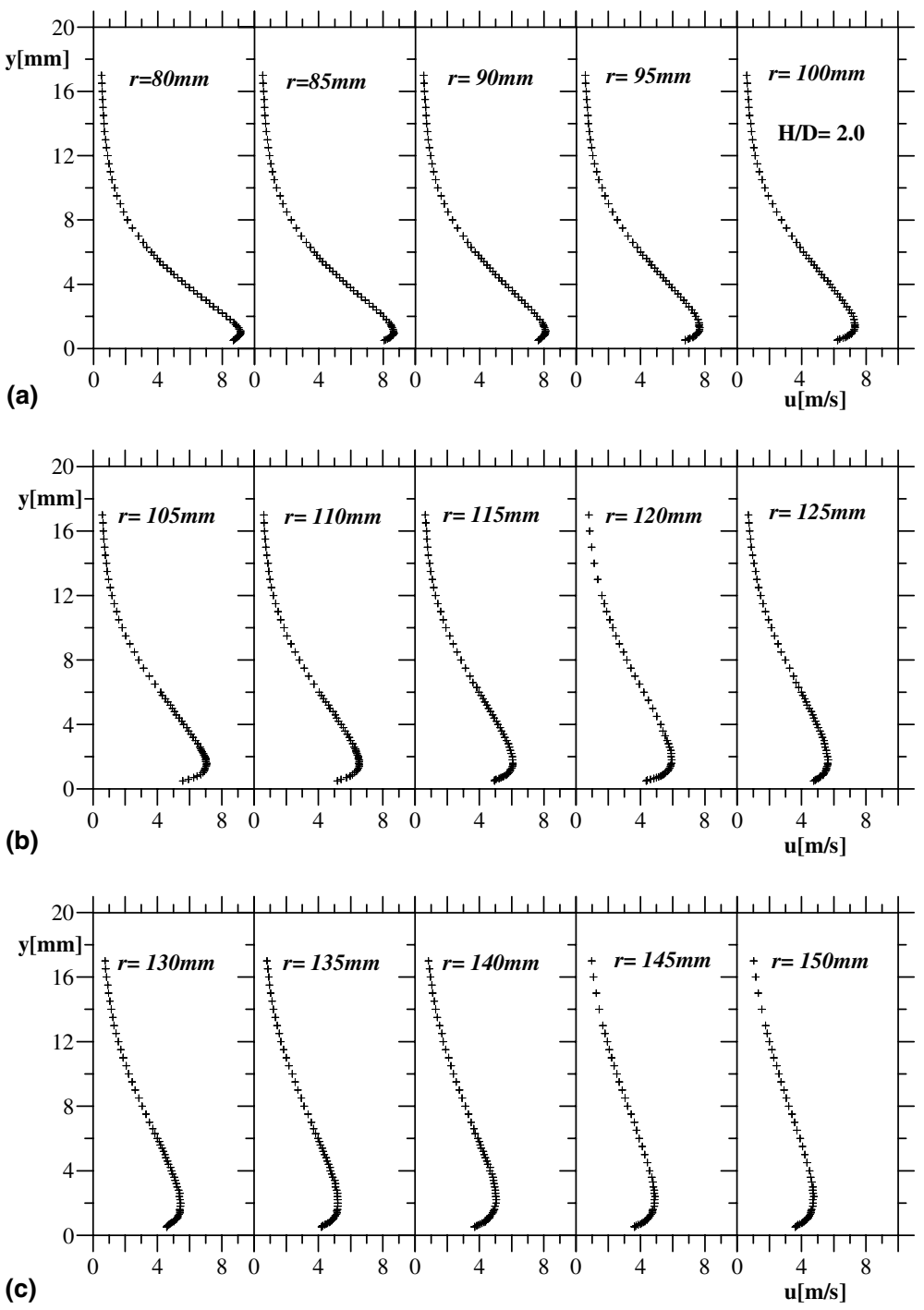

Fig. 3. Mean velocity profiles. (a) Stations $80-100 \mathrm{mmm}$, (b) stations $105-125 \mathrm{~mm}$, and (c) stations $130-150 \mathrm{~mm}$. 

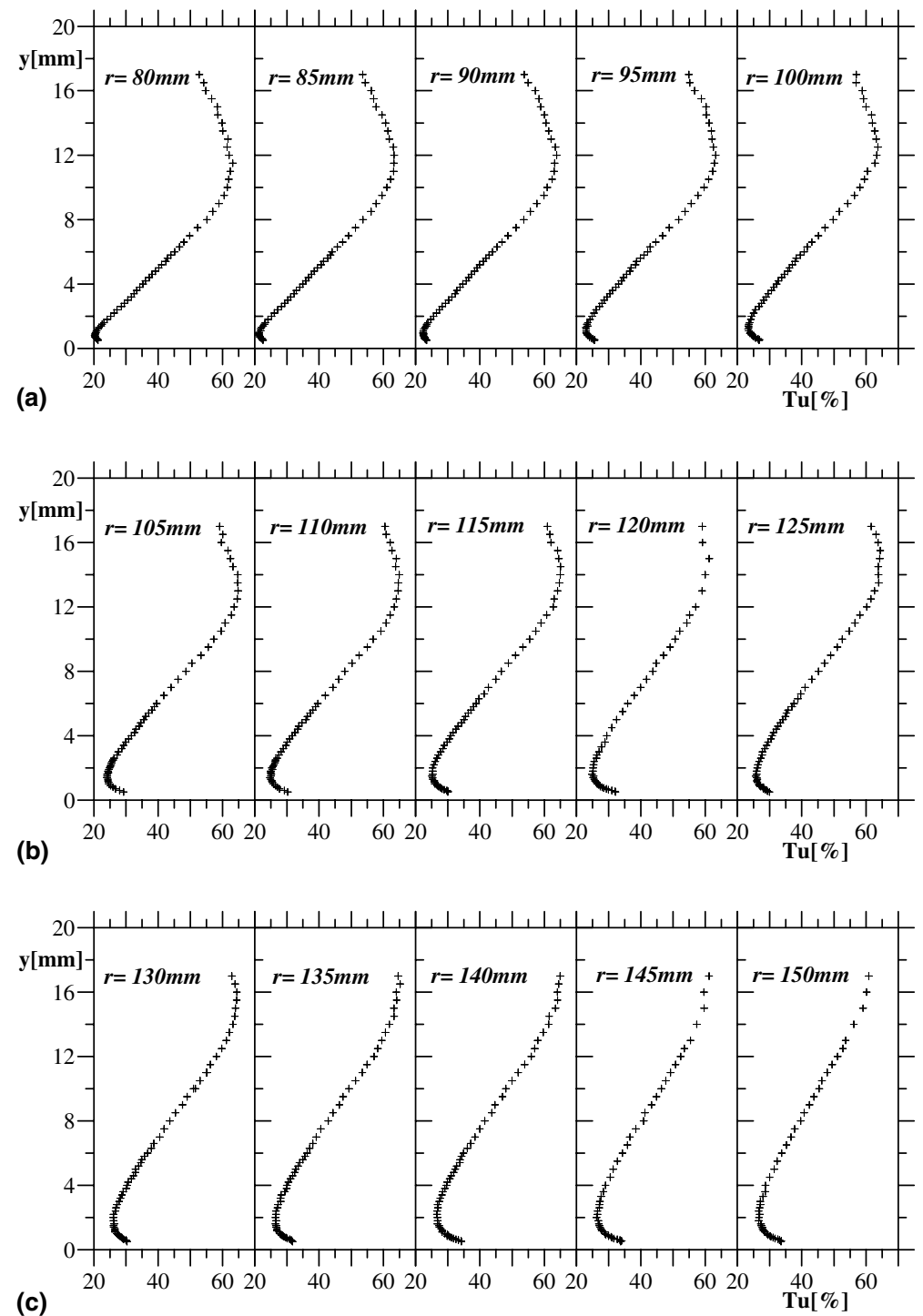

Fig. 4. Longitudinal turbulent intensity profiles. (a) Stations $80-100 \mathrm{~mm}$, (b) stations $105-125 \mathrm{~mm}$, and (c) stations $130-150 \mathrm{~mm}$. $T u[\%]=\sqrt{\overline{u^{\prime 2}}} / u$.

appears to constitute a considerable part of the inner boundary layer, and, if the outer edge of the equilibrium layer is attached to the point of maximum velocity, which is very close to the wall, then, this maximum, $u_{\mathrm{M}}$, should be an appropriate velocity scale. The conclusion, therefore, is that parameter $A$ is not invariant but changes with a deviation function.

To describe $A$, these authors proposed a simple relation of the form

$A=f\left(\frac{u_{\mathrm{M}}}{u_{\tau}}\right)$

where $u_{\mathrm{M}}$ denotes the maximum velocity for a given velocity profile.
Wygnanski et al. [7] remarked that, for a turbulent wall jet, the velocity profile cannot be universally represented in wall coordinates, as it can in the boundary layer. That is due to large variations in the additive constant in the law of the wall. In fact, depending on the jet Reynolds number, logarithmic fits can be found to their data in regions defined by specific limits. These fitted straight lines have levels varying from 5.5 to 9.5 . The existence of a well defined logarithmic region is particularly important for the determination of the skin-friction. Wygnanski et al. further remark that in previous experiments the skin-friction was either directly assessed through floating drag balances or indirectly by wall heat transfer devices or by impact probes like Stanton probes 

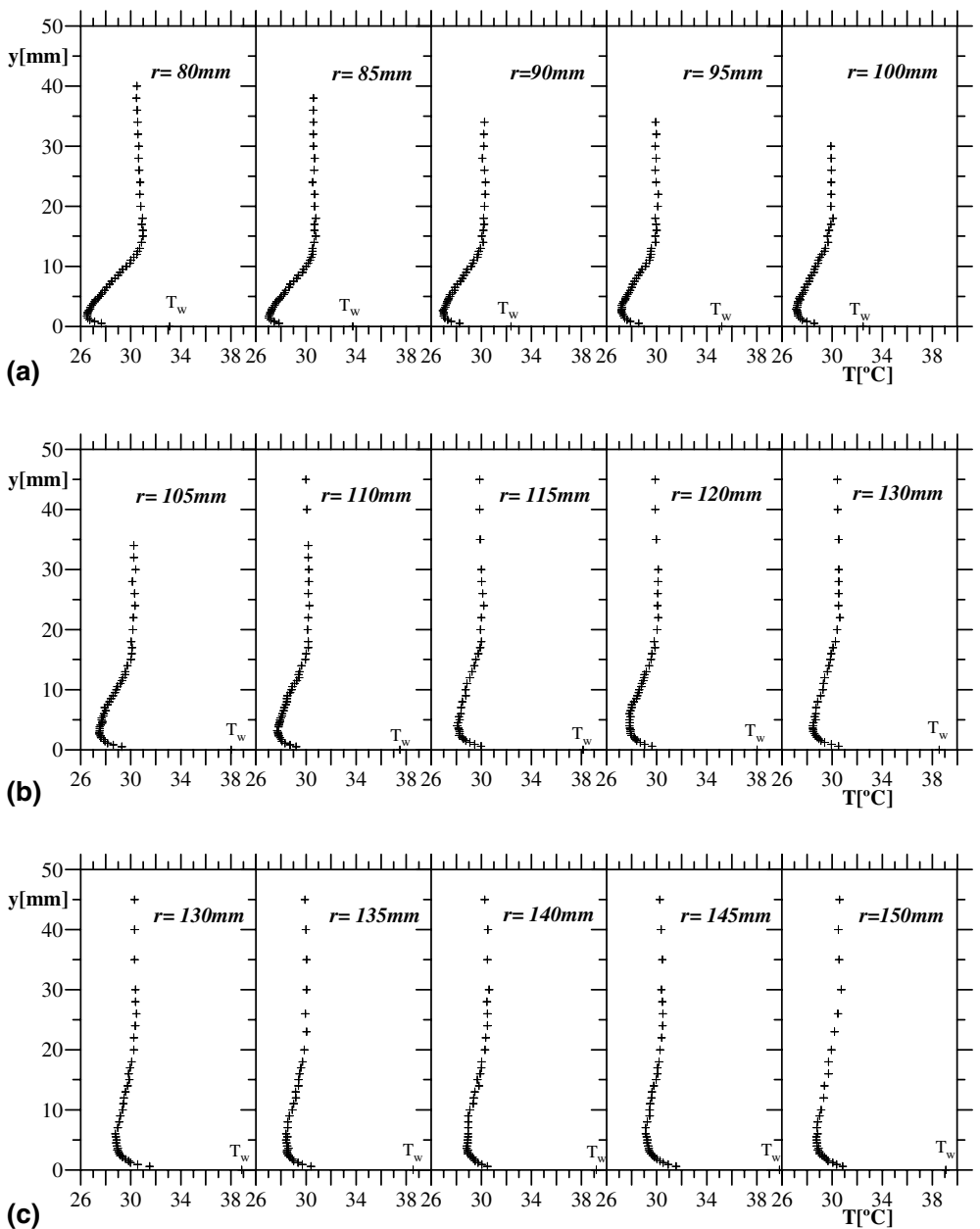

Fig. 5. Mean temperature profiles. (a) Stations $80-100 \mathrm{~mm}$, (b) stations 105-125 mm, and (c) stations 130-150 mm.

or Preston tubes. Since these devices are calibrated taking as reference the universal law of the wall, they cannot be reliably used in regions where the existence of the law of the wall can be questioned. Wygnanski et al. estimated the skin-friction through three different techniques: a momentum integral method, the mean velocity gradient in the viscous sub-layer, and by use of a Preston tube.

The establishment of the above concepts for the velocity field clearly raises some questions for the temperature field. An immediate question concerns the existence of an appropriate temperature scale at the outer edge of the equilibrium layer. At the point of velocity maximum, Fig. 5 shows that the temperature profiles reach a minimum. Thus, drawing an analogy to the velocity analyses of Narasimha et al. [6] and of Özdemir and Whitelaw [5], one would expect the appropriate scaling temperature parameter to be this minimum temperature.
The law of the wall for the temperature profile can be written as

$\frac{T_{\mathrm{w}}-T}{t_{\tau}}=\frac{1}{k_{t}} \ln \left(\frac{y u_{\tau}}{v}\right)+B$

where $t_{\tau}$ is the friction temperature and $\kappa_{t}$ is the von Karman constant for the temperature field.

The expected parametric behavior of $B$ is then to be represented by

$B=g\left(\frac{T_{\mathrm{w}}-T_{\mathrm{m}}}{t_{\tau}}\right)$

where $T_{\mathrm{w}}$ represents the wall temperature, $T_{\mathrm{m}}$ the minimum temperature in a given profile and $t_{\tau}$ is the friction temperature.

To find the values of $A$ and of $B$, the graphical method of Coles [42] was used. Here, we must point out that the thickness of the inner turbulent region for an impinging jet is very thin, so that the fitting of a straight line to the 
fully turbulent region is a difficult affair. For a plane wall jet, the fully turbulent region is rather arbitrary [8], normally being located within the interval $70<y u_{\tau} / v<170$. Since the analysis of Wygnanski et al. [7] suggests that von Karman's parameter can be considered constant and that $A$ varies from 5.5 to 9.5 , the fitting of a straight line to the velocity and to the temperature data in semilog plots in the region $70<y u_{\tau} / v<170$ can then be used to find $u_{\tau}, A, t_{\tau}$ and $B$ in Eqs. (4)-(7). The graphical method used for the determination of parameters $A$ and $B$ is illustrated in Fig. 6.

The resulting linear behavior of parameters $A$ and $B$ is shown in Fig. 7. This figure indicates that both $A$ and $B$ increase as the maximum jet velocity increases and the minimum jet temperature decreases, respectively. Specifically, the following equations result:

$A=1.124\left(\frac{u_{\mathrm{M}}}{u_{\tau}}\right)-27.538$

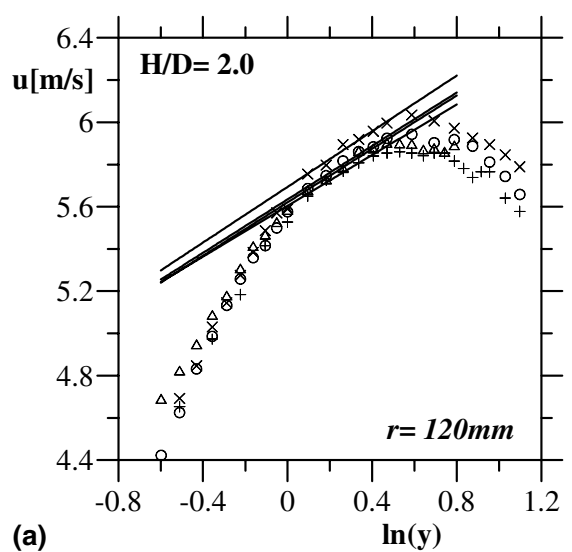

$B=1.031\left(\frac{T_{\mathrm{w}}-T_{\mathrm{m}}}{t_{\tau}}\right)-25.869$.

Thus, the trend observed by Özdemir and Whitelaw [5] is confirmed here. Furthermore, the present analysis gives us a strong hint that a possible linear behavior of $A$ and of $B$ as a function of the maximum jet velocity and of the minimum jet temperature would be in order.

When the profile-shift parameters $A$ and $B$ are subtracted from the velocity and the temperature profiles, the resulting curves exhibit the behavior of equilibrium layers that extends to the locations of the velocity maximum and the temperature minimum, respectively. This is shown in Fig. 8.

Despite our brief account of the problem of an orthogonal jet impinging on a wall, the following findings are remarkable: (1) the variation of both $A$ and $B$ is well defined and is in accordance with the account of other authors, (2) the level in the logarithmic expressions for the laws of the wall have a tendency to in-

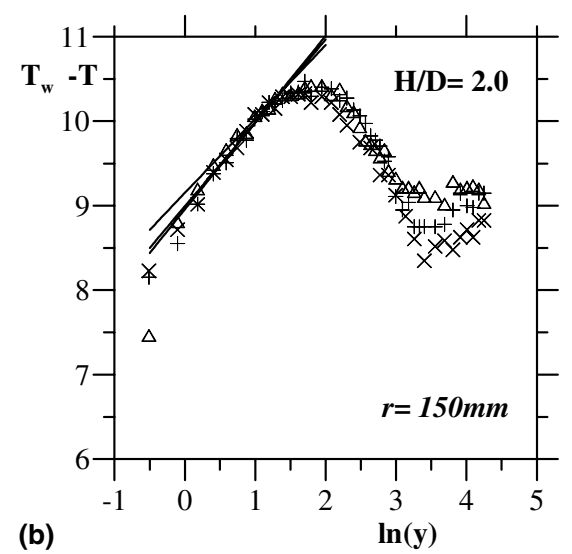

(b)

Fig. 6. Graphical method for the determination of parameters $A$ and $B$. (a) Determination of $A$ and (b) determination of $B$.
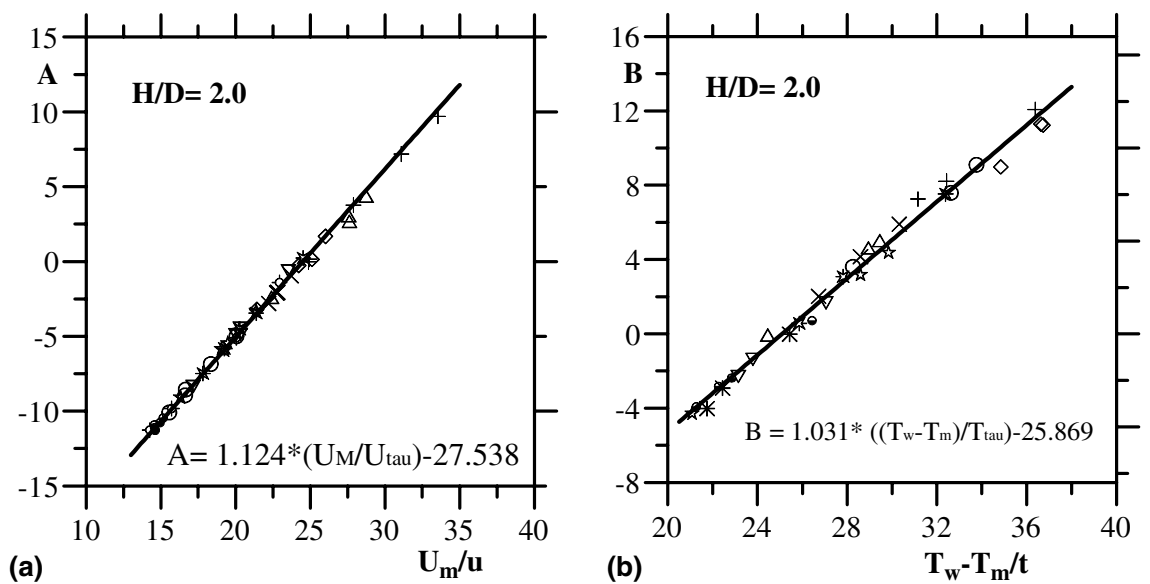

Fig. 7. Deviation function for the (a) velocity and the (b) temperature profiles. 

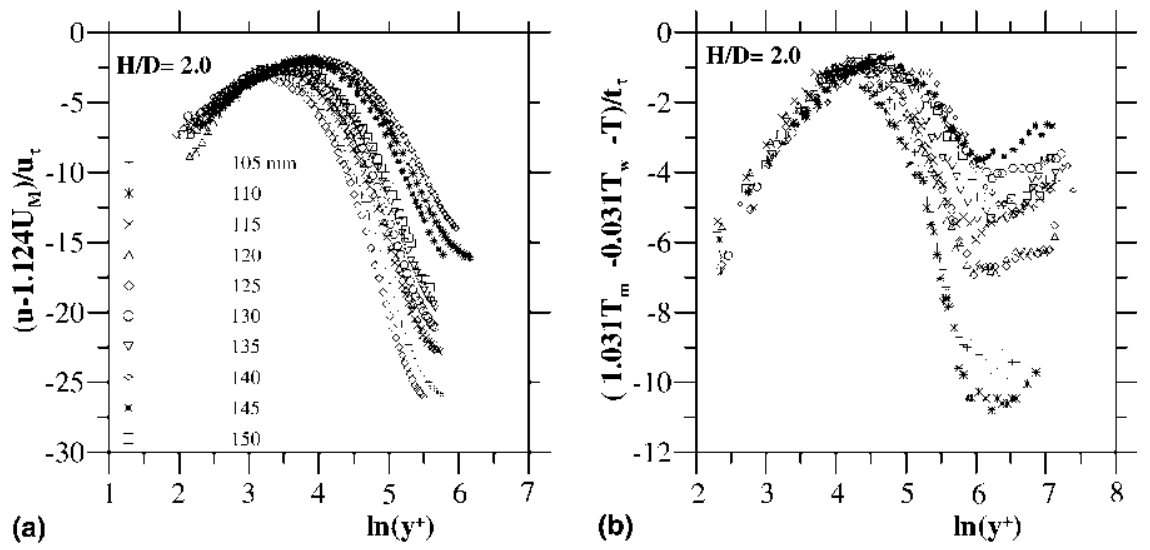

Fig. 8. Velocity and temperature profiles in inner variables, $y^{+}=y u_{\tau} / v$, with subtraction of the profile-shift parameters. (a) Velocity profiles and (b) temperature profiles.

creased with increasing maximum jet velocity and with decreasing minimum temperature.

The relations for $A$ and for $B$ derived here are particularly important for the determination of the skin-friction coefficient and of the heat transfer coefficient. This issue will be dealt with in separate article.

\section{Conclusion}

The present work has described the behavior of a semi-confined impinging jet over a heated flat plate. Experimental data for the pressure distribution, velocity and temperature fields were obtained. The heat transfer data confirmed the existence of a minimum in temperature profile away from the wall. The existence of a velocity and a temperature equilibrium layer was also investigated. The results found at this investigation indicate that the level of the logarithmic portion of the velocity and the temperature laws of the wall increases with increasing maximum jet velocity and decreasing minimum temperature. This fact, for the temperature profiles, has been observed for the first time in the course of the present research.

The present research is particularly relevant due to its application for the development of methods that can be used for the determination of the local skin-friction and of the local heat transfer coefficient.

\section{Acknowledgements}

DRSG is grateful to CAPES (Brazilian Ministry of Education) for the award of a D.Sc. scholarship in the course of the research. APSF is grateful to the Brazilian National Research Council (CNPq) for the award of a research fellowship (Grant No. 304919/2003-9). The work was financially supported by CNPq through Grant No. 472215/2003-5 and by FAPERJ through Grants E-26/171.198/2003 and E-26/152.368/2002. JS has also benefited from a CNPq research fellowship (Grant No. 550780/2002-5).

\section{References}

[1] R.P. Patel, Self preserving two dimensional turbulent jets and wall jets in a moving stream, M.Sc. Thesis, McGill University, Montreal, 1962.

[2] A. Tailland, J. Mathieu, Jet parietal, J. Mecanique 6 (1967) 1.

[3] V. Ozarapoglu, Measurements in incompressible turbulent flows. D.Sc Thesis, Laval University, Quebec, 1973.

[4] H.P.A.H. Irwin, Measurements in a self-preserving plane wall jet in a positive pressure gradient, J. Fluid Mech. 61 (1973) 33-63.

[5] I.B. Ozdemir, J.H. Whitelaw, Impingement of an axisymmetric jet on unheated and heated flat plates, J. Fluid Mech. 240 (1992) 503-532.

[6] R. Narasimha, K.Y. Narayan, S.P. Pathasarathy, Parametric analysis of turbulent wall jets in still air, Aeronaut. J. 77 (1973) 335.

[7] I. Wygnanski, Y. Katz, Horev, On the applicability of various scaling laws to the turbulent wall jet, J. Fluid Mech. 234 (1992) 669-690.

[8] G.P. Hammond, Complete velocity profile and "optimum" skin-friction formulas for the plane wall-jet, J. Fluids Eng. 104 (1982) 59-66.

[9] D.B. Spalding, A single formula for the law of the wall, ASME J. Appl. Mech. 28 (1961) 455-458.

[10] M. Behnia, S. Parneix, P.A. Durbin, Prediction of heat transfer in an axisymmetric turbulent jet impinging on a flat plate, Int. J. Heat Mass Transfer 41 (1998) 1845-1855.

[11] M. Behnia, S. Parneix, Y. Shabany, P.A. Durbin, Numerical study of turbulent heat transfer in confined and unconfined impinging jets, Int. J. Heat Fluid Flow 20 (1999) 1-9. 
[12] M.M. Gibson, R.D. Harper, Calculation of impinging-jet heat transfer with the low-Reynolds-number $q-\zeta$ turbulence model, Int. J. Heat Fluid Flow 18 (1997) 80-87.

[13] D.O.A. Cruz, A.P. Silva Freire, On single limits and the asymptotic behavior of separating turbulent boundary layers, Int. J. Heat Mass Transfer 41 (1998) 2097-2111.

[14] D.O.A. Cruz, A.P. Silva Freire, Note on a thermal law of the wall for separating and recirculating flows, Int. J. Heat Mass Transfer 45 (2002) 1459-1465.

[15] E. Forthmann, Uber turbulente strahlausbreitung, Ing. Arch. 5 (1934) 42.

[16] A. Sigalla, Measurements of a skin-friction in a plane turbulent wall jet, J.R. Aero. Soc. 62 (1958) 873.

[17] M.D. Fox, M. Kurosaka, L. Hedges, K. Hirano, The influence of vortical structures on the thermal fields of jets, J. Fluid Mech. 255 (1993) 447-472.

[18] D. Cooper, D.C. Jackson, B.E. Launder, G.X. Liao, Impinging jet studies for turbulence model assessment-I. Flow-field experiments, Int. J. Heat Mass Transfer 36 (1993) 2675-2684.

[19] T.J. Craft, L.J.W. Graham, B.E. Launder, Impinging jet studies for turbulence model assessment-II. An examination of the performance of four turbulence models, Int. J. Heat Mass Transfer 36 (1993) 2685-2697.

[20] K. Knowles, Computational studies of impinging jets using $\kappa-\varepsilon$ turbulence models, Int. J. Numer. Meth. Fluids 22 (1996) 799-810.

[21] D.W. Colucci, R. Viskanta, Effect of nozzle geometry on local convective heat transfer to a confined impinging air jet, Exp. Therm. Fluid Sci. 13 (1996) 71-80.

[22] M. Dianat, M. Fairweather, W.P. Jones, Predictions of axisymmetric and two-dimensional impinging turbulent jets, Int. J. Heat Fluid Flow 17 (1996) 530-538.

[23] J.-J. Shu, G. Wilks, Heat transfer in the flow of a cold, two-dimensional vertical liquid jet against a hot, horizontal plate, Int. J. Heat Mass Transfer 37 (1996) 3367-3379.

[24] C. Meola, G.M. Carlomagno, Influence of shear layer dynamics on impingement heat transfer, Exp. Therm. Fluid Sci. 13 (1996) 29-33.

[25] T. Liu, J.P. Sullivan, Heat transfer and flow structures in an excited circular impinging jet, Int. J. Heat Mass Transfer 39 (17) (1996) 3695-3706.

[26] K. Nishino, M. Samada, K. Kasuya, K. Torii, Turbulence statistics in the stagnation region of an axisymmetric impinging jet flow, Int. J. Heat Fluid Flow 17 (1996) 193-201.

[27] S. Ashforth-Frost, K. Jambunathan, Numerical prediction of semi-confined jet impingement and comparison with experimental data, Int. J. Numer. Meth. Fluids 23 (1996) 295-306.

[28] J.-Y. San, C.-H. Huang, M.-H. Shu, Impingement cooling of a confined circular air jet, Int. J. Heat Mass Transfer 40 (6) (1997) 1355-1364.

[29] K. Knowles, M. Myszko, Turbulence measurements in radial wall-jets, Exp. Therm. Fluid Sci. 77 (1998) 71-78.

[30] A.A. Kendoush, Theory of stagnation region heat and mass transfer to fluid jets impinging normally on solid surfaces, Chem. Eng. Process. 37 (1998) 223-228.

[31] J. Lee, S.-J. Lee, Stagnation region heat transfer of a turbulent axisymmetric jet impingement, Exp. Heat Transfer 12 (1999) 137-156.

[32] E. Baydar, Confined impinging air jet at low Reynolds numbers, Exp. Therm. Fluid Sci. 19 (1999) 27-33.

[33] J. Lee, S.-J. Lee, The effect of nozzle aspect ratio on stagnation region heat transfer characteristics of elliptic impinging jet, Int. J. Heat Mass Transfer 43 (2000) 555575.

[34] S.D. Hwang, C.H. Lee, H.H. Cho, Heat transfer and flow structure in axisymmetric impinging jet controlled by vortex pairing, Int. J. Heat Fluid Flow 22 (2001) 293-300.

[35] Y. Guo, D.H. Wood, Measurements in the vicinity of a stagnation point, Exp. Thermal Fluid Sci. 25 (2002) 605614.

[36] Y.M. Chung, K.H. Luo, N.D. Sandham, Numerical study of momentum and heat transfer in unsteady impinging jets, Int. J. Heat Fluid Flow 23 (2002) 592-600.

[37] T.H. Park, H.G. Choi, J.Y. Yoo, S.J. Kim, Stream line upwind numerical simulation of two-dimensional confined impinging slot jets, Int. J. Heat Mass Transfer 46 (2003) 251-262.

[38] M. Angioletti, R.M. Di Tommaso, E. Nino, G. Ruocco, Simultaneous visualization of flow field and evaluation of local heat transfer by transitional impinging jets, Int. J. Heat Mass Transfer 46 (2003) 1703-1713.

[39] V. Narayanan, J. Seyed-Yagoobi, R.H. Page, An experimental study of fluid mechanics and heat transfer in an impinging slot jet flow, Int. J. Heat Mass Transfer 47 (2004) 1827-1845.

[40] D.W. Zhou, S.-J. Lee, Heat transfer enhancement of impinging jets using mesh screens, Int. J. Heat Mass Transfer 47 (2004) 2097-2108.

[41] S.J. Kline, The purpose of uncertainty analysis, J. Fluids Eng. 107 (1985) 153-160.

[42] D. Coles, The law of the wake in a turbulent flow, J. Fluid Mech. 1 (1956) 191. 